\title{
Suicidal expressions among young people in Nicaragua and Cambodia: a cross-cultural study
}

\author{
Claudia Obando Medina', Bhoomikumar Jegannathan², Kjerstin Dahlblom³ and Gunnar Kullgren ${ }^{4 *}$
}

\begin{abstract}
Background: Whereas prevalence of suicidal expressions among young people is fairly similar in different countries, less is known about associated risk factors. This study compares young people in Nicaragua and Cambodia to examine if the pattern of association between mental health problems and suicidal expressions differs.
\end{abstract}

Methods: 368 and 316 secondary school students, from each country respectively, participated. Self-reported suicidal expressions, exposure to suicidal behavior in significant others and mental health problems among the students were measured using Attitude Towards Suicide (ATTS) and the Youth Self-Report (YSR) questionnaires.

Results: Prevalence of serious suicidal expressions (plans and attempts) during recent year, did not differ between countries. Cambodian young people scored significantly higher on all eight YSR-syndromes, except for withdrawn/ depressed. In Nicaragua, all YSR-syndromes were significantly associated with serious suicidal expressions in both genders compared to Cambodia where only one syndrome showed an association in each gender; Withdrawn/ depressed among girls and Somatic complaints among boys. Associations between being exposed to suicide among significant others and serious suicidal expressions also differed between Cambodia and Nicaragua.

Conclusions: While the magnitude of serious suicidal expressions is similar between these structurally similar but culturally different countries, determinants behave differently. Qualitative studies are warranted to further explore cultural specific determinants for suicidal expressions among young people.

Keywords: Suicidal expressions, Adolescents, Young People Cross-cultural comparison, Nicaragua and Cambodia

\section{Background}

Understanding suicide attempts and other related suicidal expressions is essential to improve mental health and to prevent suicide among young people. A review of population based studies estimated that a mean proportion of $9.7 \%$ of adolescents report a life-time suicide attempt [1]. Even though not all attempts are motivated by a wish to die the attempt still represents the strongest single risk factor for a suicide and by its own is a strong indicator of severe mental distress $[2,3]$

Background and meaning of suicidal expressions differ between cultures and cross-cultural studies leads to a deeper understanding of suicidal expressions among young people [3]. For example, socio-economic factors

\footnotetext{
* Correspondence: gunnar.kullgren@psychiat.umu.se

${ }^{4}$ Department of Clinical Sciences, Division of Psychiatry, Umeå University, SE90187 Umeå, Sweden Full list of author information is available at the end of the article
}

or mental health problems influence suicidal behavior differently in different countries $[4,5]$ and religion might be a protective factor for suicidal expressions in some countries and in others not [6].

Furthermore, the gender differences in suicidal expressions must be taken into consideration in cross-cultural studies. For completed suicides, there is a male dominance in most countries with exception in some Asian countries $[7,8]$ but for suicide attempts and other suicidal expressions the gender pattern is different and more complex. In hospital-based samples, girls predominate whereas community based samples show few gender differences. For example, a Nicaraguan hospital based survey showed that suicide attempts were more than three times as common among girls as compared to boys [9], whereas a community-based study in the same setting showed no significant gender differences in self-reported suicide attempts [10]. Gendered cultural beliefs
C Biomed Central 
influencing help seeking behavior might be one explanation, similar to what has been reported for young American Indians [11].

Most cross-cultural studies have been from middle or high-income countries $[4,12]$ and to our knowledge there is no study that compares suicidal expressions among young people in relation to established risk factors such as mental health problems and exposure to suicide between low-income countries from different continents

This study compares Nicaragua and Cambodia, two countries that have been through civil war, extreme social and political turmoil and share common structural conditions and widespread extreme poverty. In Nicaragua, suicide rates among young people are the highest among all Latin and Central American countries [13]. There are no reliable figures on suicide rates in Cambodia. Despite these structural similarities, there are also cultural differences. By comparing these two countries, we hope to throw new light on suicidal expressions and their determinants among young people.

\section{Method}

\section{Definitions}

The nomenclature in the field of suicidology "perennially dissatisfies researchers and clinicians", as De Leo and co-authors put it in their overview article [14]. In the present study, we use a term that most researchers seem to be satisfied with-suicidal expressions which cover a spectrum of suicide related phenomena except completed suicide. It includes life weariness, death thoughts, death wishes, suicide ideation, suicide plans and suicide attempts.

\section{The settings}

Nicaragua and Cambodia share similar demographic characteristics and both are ranked as "Medium human development" countries in the Human Development Index 2010 (117 and 126 rank respectively). Though Nicaragua is located in Central America and Cambodia in Southeast Asia, both are located in the tropical zone between 10 and 15 degrees north of the equator.

Poverty is a major concern as $16 \%$ of the population in Nicaragua and 37\% in Cambodia live under absolute poverty (< 1 USD per day) $[15,16]$. Cambodia is less urbanized as $80 \%$ of the population live in rural areas when compared to $41 \%$ in Nicaragua.

In both countries the population is young. In Nicaragua $35 \%$ (median age 22) of the population of 5.7 million and 33\% of 14.8 million in Cambodia are below 15 years (median age 22) $[17,18]$.

Adult literacy rates are estimated to be $78 \%$ in both countries, and mean years of schooling are around 6 years [19]. In Nicaragua, the level of education remains the lowest in Central America and $72 \%$ of the populations do not complete secondary education. Eighty percent of students attend public schools. Drop-out rates are high, and it takes an average of 10 years to complete 6 years of primary school [20].

In Cambodia, the formal educational structure consists of six years of primary school, three years of secondary school, and three years of higher secondary school. For every one thousand students who enroll in primary school, only 27 graduate from upper secondary school [21].

Young people in Nicaragua and Cambodia face similar health challenges, such as sexual abuse, drug abuse and reproductive health problems-all factors correlating with suicidal behavior [22].

Both Cambodia and Nicaragua suffered violence and social turmoil but of different nature. In Cambodia, the trauma of the 'Pol Pot era' has left scars in the parents of today's young people. Nicaragua has likewise had a history of political instability and civil war but without the extreme collective trauma that characterizes the Cambodian situation [23].

In both countries, family relationships are highly valued and include relatives beyond the nuclear family unit even though family structure in Nicaragua is more dismantled, much due to migration [24].

The most obvious difference is religion. In Nicaragua, Catholicism strongly dominates whereas Buddhism is the predominant religion in Cambodia

\section{Instruments}

The Attitudes towards Suicide (ATTS) is a self-report questionnaire that covers three areas; (1) Exposure to suicide from significant others such as parents, siblings, friends etc., (2) Attitudes towards suicide and (3) own suicidal expressions. In the present study we used sections 1 and 3 . The psychometric properties of the instrument have been reported in previous studies [25-27]. The semi-structured questionnaire was translated to Spanish and Khmer for use in Nicaragua and Cambodia, respectively. The translated version of ATTS was discussed among professionals and field-tested, and had been used in both the countries, previously $[10,28,29]$.

In this study 'Exposure to suicide from significant others' was defined as reported attempted or completed suicide among parents, siblings, partners or friends. Regarding own suicidal expressions, 'Serious suicidal expression' was defined as either plans or attempts during recent year.

The Youth Self-Report (YSR) developed by Achenbach and colleagues is a self-report questionnaire for 11-18 year olds that evaluates competencies and behavioral problems. In our study we explored the 
behavioral part, consisting of 112 items with statements of behaviors or symptoms, including 16 items indicating social desirability. The participants responded as following: $0=$ not true; $1=$ somewhat or sometimes true; and $2=$ very true or often true. The items are combined into eight syndromes: withdrawn/ depressed, somatic complaints, anxious/depressed (together constituting the internalizing syndrome), rule breaking behavior and aggressive behavior (together constituting the externalizing syndrome), social problems, thought problems and attention problems [25]. The total problems score is the sum of all the responses comprising of the different syndromes in YSR, whose reliability and validity has been established across diverse cultural settings $[10,12,30]$.

\section{Study design and sample}

We conducted a cross-sectional study among students aged 15-18 years in public schools in both countries during the year 2008-2009.

\section{Nicaragua}

This study was conducted in the nine public urban secondary schools in Leon municipality where 3162 pupils were enrolled in school during the study period. We used a random stratified sampling technique proportional to the size of each school to select the informants. For the final analyses 188 boys and 180 girls were included.

\section{Cambodia}

A sample of all the students of randomly selected classes, from level 10 and 11 in the two higher secondary schools took part in the study. The sample size, depending on the size of the class, was arrived at based on the prevalence figures from previous studies in Nicaragua [10]. The two higher secondary schools are located in Takhmau, Kandal province, a semi-urban area, $8 \mathrm{~km}$ south-east of Phnom Penh, the capital city of Cambodia. For the final analyses 151 boys and 165 girls were included.

\section{Ethical considerations}

The study was approved by the ethical committee of the UNAN León University, Nicaragua and Umeå University (07-046 M), Sweden. In Cambodia the study was approved by the school-directors and the parents were informed about the study through the parent-teacher association. In both settings, we visited each school and explained the aims of the study to the students as well as to the principals, teachers and the representatives of the educational system. The participation was entirely voluntary and questionnaires were returned anonymously. The researchers informed the participants about the availability of free-counseling services.

\section{Internal consistency and social desirability}

The internal consistency was acceptable for all YSR syndromes in both countries except for attention problems among the girls in Cambodia. For internalizing and externalizing syndromes the reliability was good (Table $1)$. Very similar reliability coefficients have been reported from, for example, a Swiss study [31].

The social desirability scale contains items like: "I am quite honest", "I like to help others when they need" and "I try to be fair towards others". There was no significant difference regarding social desirability score between Cambodian and Nicaraguan youth.

\section{Analysis}

The missing data on single YSR-items was less than 10\% in both countries and the missing values were imputed by the median. Chi -square was used to compare prevalence by country and gender and t- tests were used to compare mean YSR values by country and gender. Uniand multivariate analyses were used to analyze the association between serious suicidal expressions vs. YSR-syndromes and exposure to suicide. Since validated cut-off points for "casesness" among YSR-syndromes do not exist for Nicaragua or Cambodia, the YSR-syndromes were entered as continuous variables in the logistic regression analyses, i.e. ORs were calculated based on one point increment on YSR-syndrome scores.

\section{Results}

The total sample size was 684 students, $54 \%$ from Nicaragua and $46 \%$ from Cambodia. $50.4 \%$ of the informants were girls and there were no significant differences in gender distribution between countries.

\section{Prevalence of suicidal expression by gender and country}

In both genders, death thoughts, death wishes and suicide ideation and suicide attempts were more frequently

Table 1 Internal consistencies (Cronbach's Alfa) for YSR syndrome scales

\begin{tabular}{lcccc}
\hline & \multicolumn{2}{c}{ Nicaragua } & \multicolumn{2}{c}{ Cambodia } \\
\hline Anxious/depressed & Boys & Girls & Boys & Girls \\
\hline Withdrawn/depressed & 0.68 & 0.76 & 0.69 & 0.72 \\
\hline Somatic complaints & 0.59 & 0.67 & 0.60 & 0.62 \\
\hline Social problems & 0.72 & 0.66 & 0.66 & 0.67 \\
\hline Thought problems & 0.66 & 0.70 & 0.67 & 0.54 \\
\hline Attention problems & 0.66 & 0.61 & 0.66 & 0.67 \\
\hline Rule breaking behavior & 0.68 & 0.70 & 0.30 & 0.56 \\
\hline Aggressive behavior & 0.72 & 0.68 & 0.60 & 0.57 \\
\hline Internalizing symptoms & 0.78 & 0.76 & 0.73 & 0.74 \\
\hline Externalizing symptoms & 0.84 & 0.85 & 0.82 & 0.84 \\
\hline
\end{tabular}


reported by young people in Nicaragua than in Cambodia. However, for serious suicidal expressions (plans and/or attempts) there were no significant differences within either gender Table 2.

Mental health syndromes (YSR) by gender and country As shown in Table 3, Cambodian adolescents scored significantly higher on almost all YSR-syndrome scales with exception of rule-breaking behavior in both genders and withdrawn depressed and externalizing syndrome among boys. Despite significant country differences, there were few gender differences within each country.

\section{Exposure to suicide from significant others by gender and country}

Total prevalence of exposure to suicide by significant others (parents, siblings, partners or friends) differed between countries. $16.8 \%$ in Cambodia and $26.4 \%$ in Nicaragua $\left(X^{2}=9.126 ; \mathrm{p}=0.00\right)$. Among girls, there were no significant difference in terms of reported exposure between countries, but Nicaraguan boys had higher exposure to suicide by significant others $(25.5 \%)$ than Cambodian boys $(12.6 \%)\left(X^{2}=8.855 ; \mathrm{p}=0.00\right)$

\section{Association between YSR-syndromes and exposure to suicide}

Table 4 shows univariate analyses within each gender divided for country on YSR-syndromes (entered as continuous variables in the logistic regression model) and exposure in relation to serious suicidal expressions. Among girls in Nicaragua all factors showed a significant association with serious suicidal expressions whereas in Cambodian girls only withdrawn/depressed, internalizing and exposure were associated. This crossnational difference was even more pronounced among boys where all factors were significantly associated with serious suicidal expressions among Nicaraguan boys but only one factor, somatic complaints, showed association among Cambodian boys.
More from a gender perspective, the pattern of association between factors and serious suicidal expressions was similar between boys and girls in Nicaragua where all factors showed a significant association with serious suicidal expressions. In Cambodia, three of the factors were significantly associated for girls, whereas boys only showed a significant association between somatic complaints and serious suicidal expression.

In multivariate analyses, performed separately for each gender and country, where all factors were entered in a logistic regression model, no significant association remained among Cambodian boys whereas anxious/ depressed $(\mathrm{OR}=1.108 ; \mathrm{p}=0.00)$ and exposure $(\mathrm{OR}=$ 4.249; $\mathrm{p}=0.01)$ remained significant among Nicaraguan boys. Among Cambodian girls only withdrawn/ depressed $(\mathrm{OR}=1.426 ; \mathrm{p}=0.00)$ remained and among Nicaraguan girls anxious $/$ depressed $(\mathrm{OR}=1.149 ; \mathrm{p}=$ $0.00)$ and somatic complaints $(\mathrm{OR}=1.390 ; \mathrm{p}=0.01)$ remained significantly associated.

\section{Discussion}

The overall aim of the present paper was to examine suicidal expressions and some possible determinants in two very poor post-conflict societies from two different continents to identify differences that might reflect cultural differences. Three findings are of particular interest.

Firstly, this study shows that there is no significant difference in prevalence of serious suicidal expressions in Cambodia as compared to Nicaragua, even though milder expressions differ somewhat. Overall in both countries, prevalence of various suicidal expressions is in the lower range as compared to studies from middle or high income countries [4,32].

Secondly, this study shows that Cambodian youth, both girls and boys, score considerably higher on almost all YSR-syndromes than Nicaraguan youth, for many syndromes with twice as high scores. Several previous studies have reported cross-cultural differences on YSRscores but mostly to a lesser extent. For example, in a

Table 2 Prevalence of confirmatory responses to questions on suicidal expressions during recent year-stratified for gender and country

\begin{tabular}{|c|c|c|c|c|c|c|}
\hline \multirow[t]{2}{*}{ Suicidal expressions } & \multicolumn{3}{|c|}{ Boys } & \multicolumn{3}{|c|}{ Girls } \\
\hline & $\begin{array}{c}\text { Nicaragua } \\
\mathrm{n}=188 \\
\%\end{array}$ & $\begin{array}{c}\text { Cambodia } \\
\mathrm{n}=151 \\
\%\end{array}$ & $\mathrm{p}$ & $\begin{array}{c}\text { Nicaragua } \\
\mathrm{n}=180 \\
\%\end{array}$ & $\begin{array}{c}\text { Cambodia } \\
\qquad \begin{array}{c}n=165 \\
\%\end{array}\end{array}$ & $\mathrm{p}$ \\
\hline Life weariness & 34.0 & 26.5 & n.s. & 34.4 & 24.8 & n.s. \\
\hline Death thought & 36.2 & 25.8 & .042 & 43.3 & 26.1 & .001 \\
\hline Death wishes & 25.0 & 14.6 & .018 & 33.9 & 17.6 & .001 \\
\hline Suicide ideation & 17.6 & 8.6 & .017 & 27.8 & 13.3 & .001 \\
\hline Plans/attempt & 10.1 & 16.6 & n.s. & 12.8 & 12.7 & n.s. \\
\hline Any expression & 57.4 & 54.3 & n.s. & 63.3 & 49.1 & .008 \\
\hline
\end{tabular}


Table 3 Mean scores, standard deviation (SD) for YSR-syndromes as related to gender and country

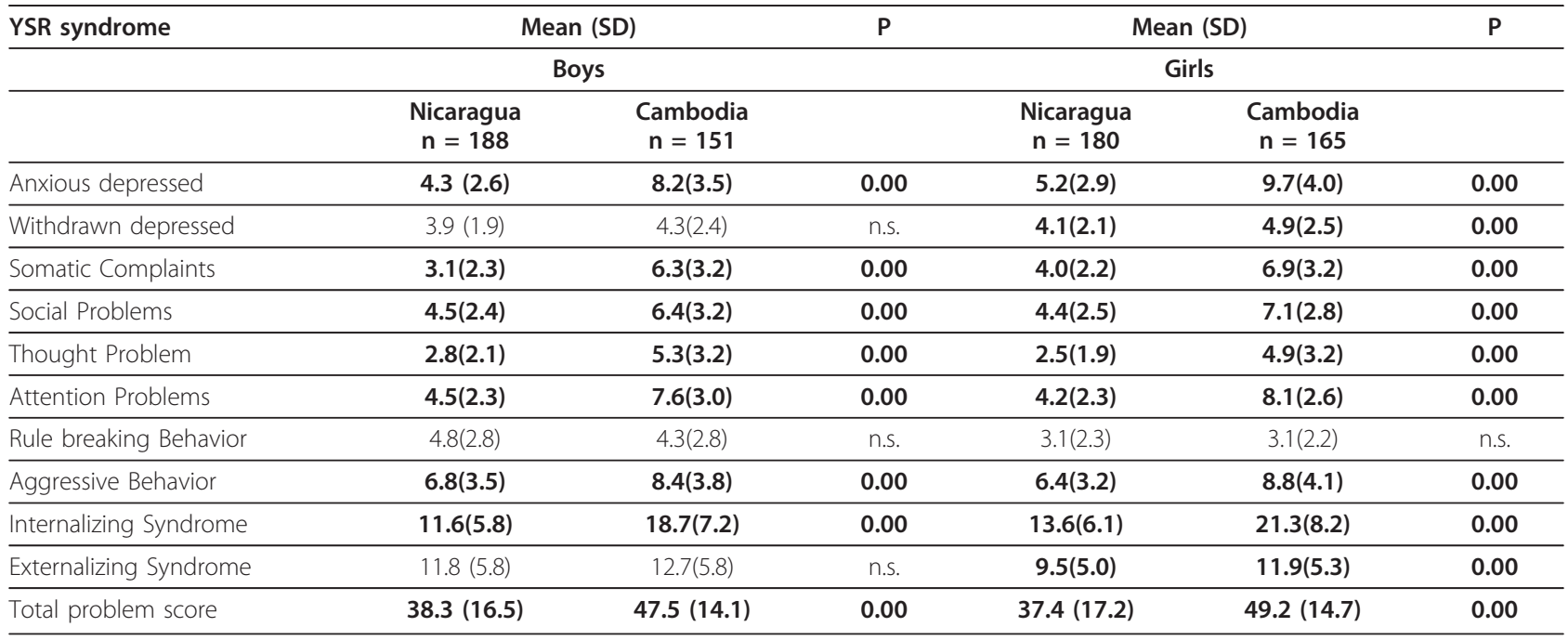

study comparing adolescents in Greece and Finland, there were higher level of anxiety and depression in Greece but the overall conclusion was that differences between these Northern and Southern regions were small [33]. A study comparing YSR-scores from seven countries also concluded differences were small between countries [34]. Being cross-sectional, our study cannot explain the reporting of higher mental distress by Cambodian youth compared with youth from Nicaragua. It is possible that the fairly recent period of collective trauma, experienced by the parents and grandparents of youth, may contribute to the mental health problems in Cambodia. Several studies have shown that mental health problems are common among the survivors of genocide and that mental health problems are likely to

Table 4 Crude ORs for YSR-syndromes and exposure to suicide among significant others as related to serious suicidal expressions

\begin{tabular}{lccccccccc}
\hline & \multicolumn{4}{c}{ Girls } & \multicolumn{4}{c}{ Boys } \\
\hline & Nicaragua & Cambodia & Nicaragua & \multicolumn{2}{c}{ Cambodia } \\
\hline & OR & $\mathbf{p}$ & OR & $\mathbf{p}$ & OR & $\mathbf{P}$ & OR & $\mathbf{p}$ \\
\hline Anxious/depressed & 1.506 & .000 & 1.086 & n.s. & 1.556 & .000 & 1.083 & n.s. \\
\hline $\begin{array}{l}\text { Withdrawn/ } \\
\text { depressed }\end{array}$ & 1.275 & .032 & 1.328 & .002 & 1.650 & .000 & 1.164 & n.s. \\
\hline Somatic complaints & 1.545 & .000 & 1.143 & n.s. & 1.248 & .028 & 1.189 & .016 \\
\hline Social problems & 1.363 & .001 & 1.053 & n.s. & 1.478 & .001 & 1.061 & n.s. \\
\hline Internalizing & 1.236 & .000 & 1.064 & .023 & 1.205 & .000 & 1.054 & n.s. \\
\hline Externalizing & 1.181 & .021 & 1.044 & n.s. & 1.156 & .001 & 1.017 & n.s. \\
\hline Total YSR problems & 1.066 & .000 & 1.032 & n.s. & 1.068 & .000 & 1.019 & n.s. \\
\hline Exposure to suicide & 2.871 & .021 & 2.793 & .040 & 6.333 & .000 & 2.000 & n.s.
\end{tabular}

(YSR-syndromes entered as continuous variables in the logistic regression analyses) be transmitted from one generation to the next through various mechanisms [35].

Thirdly, mental distress as related to suicidal expressions showed a different pattern in Cambodia and Nicaragua. Despite higher level of mental distress in Cambodia, the prevalence of serious suicidal expressions was not different between the two countries. Furthermore, almost all mental syndromes were strongly associated with serious suicidal expressions in Nicaragua but few associations were identified in Cambodia. We believe that cultural differences between these two countries with otherwise similar socio-economic conditions might play a role to prevent high level of mental distress from increasing serious suicidal expressions. Religion has been suggested to act as a protective factor, even though some studies show a mixed picture $[6,36]$ Despite the fact that the Catholic church in Nicaragua strongly condemns suicidal behavior whereas Buddhism seems to have a more tolerant view, serious suicidal expressions are as common in Nicaragua despite lower mental distress. One explanation could be that Nicaraguan culture is more secularized than Cambodia where traditional values still have a stronger influence on young people.

Fourthly, there are gender and country differences, where Nicaraguan boys exposed to suicide were almost nine times more likely to report own serious suicidal expressions while there was no significant association for Cambodian boys. Association between exposure to suicide and own suicidal expressions have been reported from many countries [1]. For example, in Nicaragua this has been confirmed in a community based study [10]. In most previous studies the association has been reported to be more evident among girls. For Nicaraguan boys depressive symptoms and exposure were associated with 
serious suicidal expressions whereas for Cambodian boys they were not.

\section{Limitations}

There are various definitions on what is meant with culture and cross-cultural research [3]. Even though this study does not take the cultural context into consideration in the analyses, we add this perspective in the interpretation of our results and we suggest that this makes it legitimate to label the study "cross-cultural".

Small sample size might have limited the results. The research instruments, ATTS and YSR, were developed in high income countries and may not measure the same constructs in the same manner in low-income countries such as Nicaragua and Cambodia. The nuances of emotions and psychological constructs are challenges to fathom particularly when the instrument is translated into different languages for cross-cultural comparison [3]. However, both instruments have been used in diverse settings and found valid $[26,27,29,30]$ ). Another important consideration is that the study deals with young people attending school and findings cannot be generalized to those outside the educational system.

\section{Conclusion}

This study lends weight to the argument that both universality as well as cultural specificity characterizes serious suicidal expressions as suggested by several researchers [37]. Whereas prevalence shows less variation between cultures, this study shows that associated factors behave differently, which we believe calls for different preventive approaches. Qualitative research approaches are warranted to further explore possible cultural bound mechanisms behind suicidal expressions among young people.

\section{Acknowledgements}

This study was supported by research grants from Swedish International Development Cooperation Agency (SIDA).

\section{Author details \\ ${ }^{1}$ Centre for Demography and Health Research, Nicaraguan National Autonomous University, León, Nicaragua. ${ }^{2}$ Center for Child and Adolescent Mental Health (CCAMH), Takhmau, Cambodia. ${ }^{3}$ Department of Public Health and Clinical Medicine, Division of Epidemiology and Global Health, Umeå University, SE-901 85 Umeå, Sweden. ${ }^{4}$ Department of Clinical Sciences, Division of Psychiatry, Umeå University, SE-901 87 Umeå, Sweden.}

\section{Authors' contributions}

CMO and BJ were involved in planning the study, organized data collection, performed analyses and drafted the manuscript. GK planned the study and supervised analyses and drafted the manuscript. KD planned the study and drafted the manuscript. All authors read and approved the final manuscript.

\section{Competing interests}

The authors declare that they have no competing interests.

Received: 7 September 2011 Accepted: 31 March 2012 Published: 31 March 2012

\section{References}

1. Evans E, Hawton K, Rodham K, Deeks J: The prevalence of suicidal phenomena in adolescents: a systematic review of population-based studies. Suicide Life Threat Behav 2005, 35:239-250.

2. Fergusson DM, Horwood $L$, Ridder EM, Beautrais AL: Suicidal behaviour in adolescence and subsequent mental health outcomes in young adulthood. J Psychol Med 2005, 35:983-993.

3. Hjelmeland $\mathrm{H}$ : Cultural research in suicidology: challenges and opportunities. Suicidology Online 2010, 1:34-52.

4. Bertolote JM, Fleischmann A, De Leo D, Bolhari J, Botega N, De Silva D, et al: Suicide attempts, plans, and ideation in culturally diverse sites: the WHO SUPRE-MISS community survey. J Psychol Med 2005, 35:1457-1465.

5. Vijayakumar $L$, John S, Pirkis J, Whiteford H: Suicide in developing countries (2): risk factors. Crisis 2005, 26:112-119.

6. Sisask M, Varnik A, Kolves K, Bertolote JM, Bolhari J, Botega NJ, et al: Is religiosity a protective factor against attempted suicide: a cross-cultural case-control study. Arch Suicide Res 2010, 14:44-55.

7. Aaron R, Joseph A, Abraham S, Muliyil J, George K, Prasad J, et al: Suicides in young people in rural southern India. Lancet 2004, 363:1117-1118.

8. Phillips MR, Li X, Zhang Y: Suicide rates in China, 1995-99. Lancet 2002, 359:835-840

9. Caldera T, Herrera A, Renberg ES, Kullgren G: Parasuicide in a low-income country: results from three-year hospital surveillance in Nicaragua. Scand J Public Health 2004, 32:349-355.

10. Rodriguez AH, Caldera T, Kullgren G, Renberg ES: Suicidal expressions among young people in Nicaragua: a community based study. SoC Psychiatry Psychiatr Epidemiol 2006, 41:692-697.

11. Freedenthal S, Stiffman A: "They might think I was crazy":Young American Indians' reasons for not seeking help when suicidal. J Adolesc Res 2007, 22:58-77.

12. Achenbach TM, Becker A, Döpfner $M$, Heiervang $E$, Roessner $V$, Steinhausen $\mathrm{HC}$, et al: Multicultural assessment of child and adolescent psychopathology with ASEBA and SDQ instruments: research findings, applications, and future directions. J Child Psychol Psychiatr 2008, 49:251-275.

13. PAHO: Health Statistics from the Americas Washington, DC; 2006 [http://new. paho.org/hq/index.php? option=com content\&task=view\&id=3148\&ltemid=259].

14. De Leo D, Burgis S, Bertolote JM, Kerkhof AJFM, Bille-Brahe U: Definitions of suicidal behavior. Lessons learned from the WHO/EURO multicentre study. Crisis 2006, 27:4-15.

15. Del Carpio X: "Voices of Nicaragua a Qualitative and Quantitative Approach to Viewing Poverty in Nicaragua". Nicaragua Poverty Assesment. Report No. 39736 The World Bank; 2007 [http://www-wds.worldbank.org/ external/default/WDSContentServer/WDSP/IB/2008/07/08/ 000333038_20080708004519/Rendered/PDF/ 397360ESWOvol110gray0cover01PUBLIC1.pdf].

16. UNPD: Human Development Reports. Multidimensional Poverty Index Geneva; 2010 [http://hdr.undp.org/en/statistics/mpi/]

17. WHO: Country information. Cambodia Geneva; 2010 [http://www.who.int/ countries/khm/en/].

18. WHO: Country reports. Nicaragua Geneva; 2010 [http://www.who.int/ countries/nic/en/].

19. UNDP: Human Development Report 2010 - 20th Anniversary Edition. The Real Wealth of Nations:Pathways to Human Development New York; 2010 [http:// hdr.undp.org/en/reports/global/hdr2010/chapters/].

20. INEC: VIII Censo de población y IV de Vivienda. Población Características Educativas Nicaragua: INEC; 2006.

21. Ministry of Education Youth and Sport: Education Sector Performance Report. Kingdom of Cambodia: Ministry of Education Youth and Sport Cambodia. Ministry of Education Youth and Sport; 2005 [http://www.moeys.gov.kh/ old_moeys.gov/en/education/espr05/espr05.pdf].

22. Dube SR, Anda RF, Felitti VJ, Chapman DP, Williamson DF, Giles WH: Childhood abuse, household dysfunction, and the risk of attempted suicide throughout the life span. JAMA 2001, 286:3089-3096.

23. Mollica RF, Poole C, Son L, Murray CC, Tor S: Effects of War Trauma on Cambodian refugee adolescents' functional health and mental health status. J Am Acad Child Adolesc Psychiatry 1997, 36:1098-1106.

24. Lancaster R: Machismo, danger and the intimacy of power in Nicaragua. Life is Hard California: University of California Press; 1992. 
25. Achenbach TM, Rescorla LA: Manual for the ASEBA School- Age Forms \& Profiles Burlington, VT: University of Vermont, Research Center for Children, Youth \&Families; 2001.

26. Arnautovska U, Grad OT: Attitudes toward suicide in the adolescent population. Crisis 2010, 31:22-29.

27. Mofidi N, Ghazinour M, Salander-Renberg E, Richter J: Attitudes towards suicide among Kurdish people in Iran. Soc Psychiatry Psychiatr Epidemiol 2008, 43:291-298.

28. Jegannathan B, Kullgren G: Gender differences in suicidal expressions and their determinants among young people in Cambodia, a post-conflict country. BMC Psychiatry 2011, 11:47.

29. Obando Medina CM, Herrera A, Kullgren G: Suicidal expression in adolescents in Nicaragua in relation to Youth Self-Report (YSR) syndromes and exposure to suicide. Clin Pract Epidemiol Ment Health 2011, 7:89-96.

30. Ivanova MY, Achenbach TM, Rescorla LA, Dumenci L, Almqvist F, Bilenberg $N$, et al: The generalizability of the Youth Self-Report syndrome structure in 23 societies. J Consult Clin Psychol 2007, 75:729-738.

31. Steinhausen HC, Metzke CW: Youth Self-Report of behavioral and emotional problems in a Swiss epidemiological study. $J$ Youth Adolesc 1998, 27:429-441.

32. Grunbaum JA, Kann L, Kinchen SA, Williams B, Ross JG, Lowry R, et al: Youth risk behavior surveillance-United States, 2001. J Sch Health 2002, 72:313-328.

33. Kapi A, Veltsista A, Sovio U, Järvelin MR, Bakoula C: Comparison of selfreported emotional and behavioural problems in adolescents from Greece and Finland. Acta Paediatr 2007, 96:1174-1179.

34. Verhulst FC, Achenbach TM, Van der Ende J, Erol N, Lambert MC, Leung PW, et al: Comparisons of problems reported by youths from seven countries. Am J Psychiatry 2003, 2003(160):1479-1485.

35. Carlsson E, Rosser-Hogan R: Cross-cultural response to trauma: a study of traumatic experiences and posttraumatic symptoms in Cambodian refugees. J Trauma Stress 1994, 7:43-58.

36. Clarke CS, Bannon FJ, Denihan A: Suicide and religiosity-Masaryk's theory revisited. Soc Psychiatry Psychiatr Epidemiol 2003, 38:502-506.

37. Mishara BL: Cultural specificity and universality of suicide: challenges for the international association for suicide prevention. Crisis 2006, 27:1-3.

\section{Pre-publication history}

The pre-publication history for this paper can be accessed here: http://www.biomedcentral.com/1471-244X/12/28/prepub

doi:10.1186/1471-244X-12-28

Cite this article as: Medina et al:: Suicidal expressions among young people in Nicaragua and Cambodia: a cross-cultural study. BMC

Psychiatry 2012 12:28.

\section{Submit your next manuscript to BioMed Central and take full advantage of:}

- Convenient online submission

- Thorough peer review

- No space constraints or color figure charges

- Immediate publication on acceptance

- Inclusion in PubMed, CAS, Scopus and Google Scholar

- Research which is freely available for redistribution

Submit your manuscript at www.biomedcentral.com/submit 